\title{
Typical Performance of Generalised Vector Channels
}

\author{
Roberto C. Alamino \\ Aston University \\ Neural Computing Research Group \\ Birmingham, B4 7ET, United Kingdom \\ Email: alaminrc@aston.ac.uk
}

\author{
David Saad \\ Aston University \\ Neural Computing Research Group \\ Birmingham, B4 7ET, United Kingdom \\ Email:d.saad@aston.ac.uk
}

\begin{abstract}
We introduce a general matrix formulation for multiuser channels and analyse the special cases of MultipleInput Multiple-Output channels, channels with interference and relay arrays under LDPC coding using methods developed for the statistical mechanics of disordered systems. We use the replica method to provide results for the typical overlaps of the original and recovered messages and discuss their implications. The results obtained are consistent with belief propagation and density evolution results but also complement them giving additional insights into the information dynamics of these channels with unexpected effects in some cases.
\end{abstract}

\section{INTRODUCTION}

The growing use of information networks has made the study of multiuser channels highly relevant, although there is still no general theory for such channels and analytical results are only known in special cases. The main difficulty is the inability to separate the information transmission into source and channel coding.

Statistical physics provides powerful methods to derive typical properties under quenched disorder of interacting systems with a large number of units [1]. Results for multiuser channels were analysed from different and complementary points of view, resulting in new insights [2], [3]. The replica method, in particular, facilitates the derivation of typical results in various cases [4], [5], [2].

Here we employ these methods to study a class of multiuser channels, which include Multiple-Input Multiple-Output (MIMO) and relay arrays, encoded with Low-Density ParityCheck (LDPC) error-correcting codes [6], [7], [8], [9].

\section{LDPC CODED VECTOR CHANNELS}

LDPC Error Correcting Codes [6] exhibit unbeatable performance, especially at high code rate. A code is defined by a binary parity-check matrix $A=\left[C_{1} \mid C_{2}\right]$, concatenating two sparse matrices known to sender and receiver, $C_{2} \in$ $\{0,1\}^{(M-N) \times(M-N)}$ (invertible) and $C_{1} \in\{0,1\}^{(M-N) \times N}$. We analyse regular codes with $K$ non-zero entries per row, $C$ per column and rate $R=N / M$. Generalisation to irregular codes is straightforward [10]. Encoding refers to the linear mapping $\mathbf{t}=G \mathbf{s}(\bmod 2)$ of a message $\mathbf{s} \in\{0,1\}^{N}$ to a codeword $\mathbf{t} \in\{0,1\}^{M}(M>N)$ by the generator matrix

$$
G=\left(\begin{array}{c}
I \\
C_{2}^{-1} C_{1}
\end{array}\right) \quad(\bmod 2), \text { such that } A G=0 \text {, }
$$

with $I$ the $N \times N$ identity matrix. To decode means to estimate the most probable $\mathbf{t}$ [10], [5].

Matrix Formulation: Consider $L$ senders encoding messages $\mathbf{s}_{i} \in\{0,1\}^{N}, i=1, \ldots, L$, by LDPC codes with independently chosen parity-check matrices $A^{i}$ into codewords $\mathbf{t}_{i} \in\{0,1\}^{M}$ sent to $O$ receivers. At time steps $\mu=1, \ldots, M, t_{1 \mu}, \ldots, t_{L \mu}$ are transmitted and corrupted by additive white Gaussian noise (AWGN). We write

$$
\underline{r}=\mathcal{S} \underline{t}+\underline{\nu}
$$

with matrices $\underline{t} \equiv\left(t_{i \mu}\right)_{L \times M}, \underline{r} \equiv\left(r_{j \mu}\right)_{O \times M}$ and channel operator $\mathcal{S}:\{0,1\}^{L \times M} \rightarrow\{0,1\}^{O \times M}$ representing the interference and intermediate processing, whose operation on $\underline{t}$ is $t_{j \mu}(\underline{t}) \equiv(\mathcal{S} \underline{t})_{j \mu}$. The AWGN is given by the matrix $\underline{\nu}=\left(\nu_{j \mu}\right)_{O \times M}$ with $\nu_{j \mu}$ distributed as $\mathcal{N}\left(0, \sigma_{j \mu}^{2}\right)$.

Statistical Physics: Decoding becomes a statistical physics problem by defining a matrix $\underline{\tau} \equiv\left(\tau_{i \mu}\right)_{L \times M}$ at the receivers that collaborate to obtain the Marginal Posterior Maximiser (MPM) estimate $\hat{t}_{i \mu}=\operatorname{sgn}\left\langle\tau_{i \mu}\right\rangle_{\mathcal{P}(\tau \mid r)}$, minimising the bit error [11], [10], where $\mathcal{P}(\underline{\tau} \mid \underline{r}) \propto \mathcal{P}(\underline{r} \mid \underline{\tau}) \mathcal{P}(\underline{\tau})$. Collaboration formally makes the receivers into a single receiver in an information theoretic sense, but the same methodology can be applied, with minimal modifications, to the case without collaboration. For convenience, we map $\underline{t} \in\{0,1\}^{L \times M}$ into $\underline{t} \in\{1,-1\}^{L \times M}$ by $x \rightarrow(-1)^{x}$.

The performance is measured by the overlaps $d_{i}=$ $M^{-1} \sum_{\mu}\left\langle t_{i \mu} \hat{t}_{i \mu}\right\rangle_{A^{1}, \ldots, A^{L}, \underline{r}, \underline{,}}$, derived from the free-energy

$$
f=-\lim _{M \rightarrow \infty} \frac{1}{\beta M L}\langle\ln Z\rangle_{A^{1}, \ldots, A^{L}, \underline{r}, \underline{t}},
$$

where the partition function (the normalisation coefficient for the probabilities [2]) is $Z=\sum_{\underline{\tau}} \exp [-\beta \mathcal{H}(\underline{\tau} ; \underline{r})]$. The Hamiltonian $\mathcal{H}(\underline{\tau} ; \underline{r})=-\sum_{j=1}^{O} \ln \mathcal{\mathcal { P }}\left(\mathbf{r}_{j} \mid \underline{\tau}\right)-\ln \mathcal{P}(\underline{\tau})$, is calculated in the thermodynamic limit $M, N \rightarrow \infty$ and finite $R$, by the replica method [1], based on an analytical continuation for $n$ in $\langle\ln Z\rangle=\lim _{n \rightarrow 0} \partial \ln \left\langle Z^{n}\right\rangle / \partial n$.

As $\mathcal{P}(\underline{\tau})$ is just the parity-check constraint and for AWGN, 


$$
\begin{aligned}
\mathcal{P}\left(\mathbf{r}_{j} \mid \underline{\tau}\right)= & \prod_{\mu=1}^{M} \mathcal{N}\left(\hbar_{j \mu}, \sigma_{j \mu}^{2}\right), \text { we have } \\
Z= & \sum_{\underline{\tau}}\left[\prod _ { i = 1 } ^ { L } \prod _ { \nu = 1 } ^ { M - N } \delta \left(\prod_{\mu=1}^{M}\left(\tau_{i \mu}\right)^{\left.\left.A_{\nu \mu}^{i}, 0\right)\right]}\right.\right. \\
& \times \exp \left[-\beta \sum_{j=1}^{O} \sum_{\mu=1}^{M} \frac{1}{2 \sigma_{j \mu}^{2}}\left(r_{j \mu}-\hbar_{j \mu}\right)^{2}\right],
\end{aligned}
$$

where $\delta$ is a Kroenecker delta. The inverse temperature $\beta$ is the ratio between the true and the assumed noise level, which is correct for $\beta=1$ (Nishimori's temperature [1]). Although we will treat only the case $\beta=1$, we will keep the general formulation as it is important theoretically in the derivation of the entropy and energies.

For memoryless channels $\left(t_{j \mu}\right.$ a function only of $\left.t_{1 \mu}, \ldots, t_{L \mu}\right)$, it is convenient to assume $\underline{\nu}$ independent of time. The replica symmetric (RS) ansatz [10] provides the saddle point equations

$$
\begin{aligned}
\hat{\pi}_{i}\left(\hat{x}_{i}\right) & =\left\langle\delta\left(\hat{x}_{i}-\prod_{l=1}^{K-1} x_{i}^{l}\right)\right\rangle_{\mathbf{x}}, \\
\pi_{i}\left(x_{i}\right) & =\left\langle\delta\left(x_{i}-h_{i}\right)\right\rangle_{\hat{\mathbf{x}}, r}, \\
h_{i} & \equiv \frac{\sum_{\tau} \tau_{i} \mathcal{E}(r, \tau) \prod_{i=1}^{L} \prod_{l=1}^{C-1}\left(1+\tau_{i} \hat{x}_{i}^{l}\right)}{\sum_{\tau} \mathcal{E}(r, \tau) \prod_{i=1}^{L} \prod_{l=1}^{C-1}\left(1+\tau_{i} \hat{x}_{i}^{l}\right)} \\
\mathcal{E}(r, \tau) & \equiv \exp \left[-\beta \sum_{j=1}^{O} \frac{1}{2 \sigma_{j}^{2}}\left(r_{j}-\hbar_{j}\right)^{2}\right],
\end{aligned}
$$

with $\tau \equiv\left(\tau_{1}, \ldots, \tau_{L}\right)$ and $r=\left(r_{1}, \ldots, r_{O}\right)$ distributed as $\mathcal{P}(r)=$ $\prod_{j=1}^{O} \mathcal{N}\left(s_{j}, \sigma_{j}^{2}\right), s_{j} \equiv \hbar_{j}(0)$. Averages $\langle\cdot\rangle_{\mathbf{x}}$ and $\langle\cdot\rangle_{\hat{\mathbf{x}}}$ are over all distributions $\pi_{i}$ and $\hat{\pi}_{i}$, respectively.

The overlaps are $d_{i}=\left\langle\operatorname{sgn} u_{i}\right\rangle_{u_{i}}, \mathcal{P}\left(u_{i}\right)=\left\langle\delta\left(u_{i}-\bar{h}_{i}\right)\right\rangle_{\hat{\mathbf{x}}, r}$ and $\bar{h}_{i}$ differs from $h_{i}$ (6) by the product on $l$ terms which extends until $C$ instead of $C-1$. The free energy can then be obtained, with the internal energy and entropy derivable from it. The ferromagnetic (FM) solution (perfect decoding) always exist, although it can be subdominant depending on the noise level. The paramagnetic solution (no decoding) appears only for very high noise.

We solve eqs. $(5,6)$ iteratively. The algorithm becomes slow as $L$ and $O$ increase; we show results for low $L, O$ values to limit the computational effort [12], [13]. For comparison, we always use $R=1 / 4(K=4, C=3)$ and $\beta=1$. If the Hamiltonian is gauge invariant [1] one can show that the solution is exact at Nishimori's temperature.

\section{MIMO CHANNEL}

If $\mathcal{S}$ is linear and the channel memoryless, then $t_{j \mu}=$ $\sum_{i=1}^{L} S_{j i} t_{i \mu}$, reducing to the MIMO channel. Although this Hamiltonian is not gauge invariant and its solution may not be $\mathrm{RS}$, the solution is the same as Belief Propagation (BP) and its macroscopic equivalent Density Evolution (DE), giving useful information on the practical decoding limit.

Single Transmitter: $L=O=1$ is the AWGN channel [10]. Fig. 1 shows the overlap for $L=1, O=2$, all transmitters

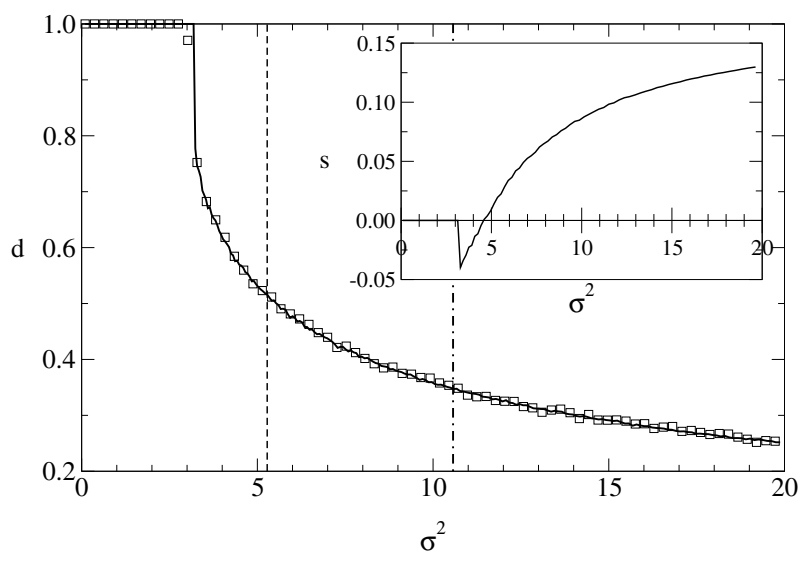

Fig. 1. Overlap for $L=1, O=2$ (solid line). Also shown are the theoretical limit for this channel (dotted-dashed) and for sending a doubled message via an AWGN channel (dashed). Squares correspond to BP with 20 random parity-matrices and $M=6000$. The inset shows the corresponding entropy. TABLE I

SHANNON'S LIMIT (SL) THE DYNAMICAL (DT) AND THERMODYNAMICAL (TT) TRANSITIONS FOR MIMO AND MAC CHANNELS. LEFT - SL FOR AN AWGN AND MIMO CHANNELS IN THE SINGLE-SENDER CASE $(L=1)$. RIGHT - RESULTS FOR THE MAC CASE $(O=1)$.

\begin{tabular}{|c|c|c|c|c||c|c|c|c|}
\hline \multicolumn{5}{|c||}{ Single Sender } & \multicolumn{4}{c|}{ MAC } \\
\hline \hline$O$ & SL $_{\text {AWGN }}$ & SL $_{\text {MIMO }}$ & DT & TT & L & SL & DT & TT \\
\hline \hline 1 & 2.41 & 2.41 & 1.59 & 2.24 & 1 & 2.41 & 1.59 & 2.24 \\
2 & 5.28 & 10.57 & 3.28 & 4.59 & 2 & 2.00 & 1.32 & 1.66 \\
3 & 8.17 & 24.50 & 4.90 & 6.68 & 3 & 1.64 & 1.24 & 1.45 \\
\hline
\end{tabular}

with power 1 and all receivers with the same $\sigma^{2}$. Up to the noise level termed the dynamical transition, the FM state is the only stable solution. It remains dominant up to the thermodynamical transition, where the non-FM state becomes dominant. Between the two, the exponential number of suboptimal (non-FM) stable solutions prevent the convergence to the FM state. Squares represent results of BP [10]; differences are due to finite size effects that disappear (slowly) with $M$.

In the inset, the entropy is zero up to the dynamical transition. Metastable suboptimal solutions, contributing to unphysical negative entropies [10], emerge above it and can be explored with a replica symmetry breaking assumption [14], [15]. The entropy crosses again the coordinate axis at the thermodynamical transition, always upper bounded by Shannon's limit (vertical dashed line).

Table I (left) compares Shannon's limit of sending the same message $O$ times via an AWGN channel (second column) with noise level equal to the MIMO channel (third column) and also the dynamical (fourth column) and thermodynamical (fifth column) transitions for $O=1,2,3$, which always occur before Shannon's limit. As expected, the more receivers, the higher the tolerated noise. However, the differences between the dynamical and the thermodynamical transitions, and between the later and Shannon's limit, increase for, in adding more receivers, the number of metastable states increases; they emerge earlier and contribute to a higher entropy.

Comparing the theoretical limit of sending a message $O$ times by an AWGN channel and by a MIMO channel with 


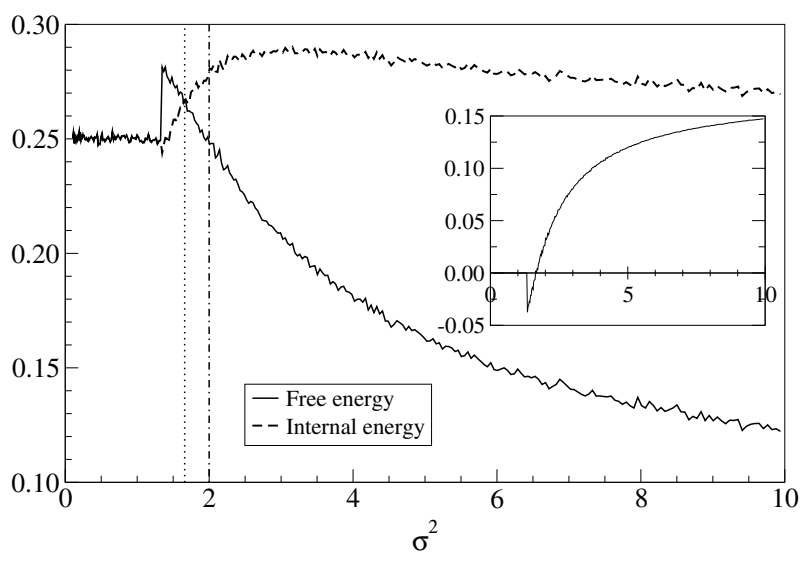

Fig. 2. Free energy and internal energy in the MAC with $L=2$ (solid and dashed lines, respectively). Also shown is the theoretical limit (dot-dashed) and the thermodynamical transition (dotted). The inset shows the entropy.

$L=1$ and $O$ receivers, the later is just $O$ times the former. This occurs because the information sent in the MIMO channel with power 1 is the same as in the $O$-replicated AWGN channel with $O$ times the power. The transitions are below the theoretical limit for the AWGN channel and significantly below the MIMO limit. This shows that in this type of channel, even with joint decoding, the available information is poorly used.

Multiple Access Channel (MAC) corresponds to the case of $O=1$. We consider again equal unit transmitting power and noise. Due to the interference, one must guarantee that all terms have the same order in $L$ and normalise the sum by $L^{-1 / 2}$.

For $L=2$, the energies are given in fig. 2. Shannon's limit is $\sigma^{2}=2$ (dot-dashed line). The point where the free energy differs from the internal energy marks the dynamical transition. The thermodynamical transition is signalled by their later crossing (dotted line).

Table I (right) shows the results for $L=1,2,3$. The second column gives Shannon's limit, showing the deterioration in performance as $L$ increases. This is also evident in the RS results for the dynamical (second column) and thermodynamical (third column) transitions. Here, the difference between both transitions decreases with $L$, meaning that additional inputs seem to increase the number of sub-optimal states (reducing their free energy and affecting the thermodynamical transition) but have a lesser effect on the onset of the metastable states.

We consider two scenarios for $L \rightarrow \infty$ : random interference, which due to the isomorphism between CDMA and MIMO, is exactly the one calculated in [16] if $S_{j i}=s_{j i} / \sqrt{L}$ with $s_{j i}$ i.i.d. with unit variance and zero odd moments, and deterministic interference, with $S$ not random. The later is of little interest as the capacity grows with $\log O$ while the transmitted information grows linearly with $L$, leading the capacity per user to zero.

\section{Channels With Interference}

Codewords are corrupted by (small) deterministic interference from all other transmitters, defined through the squared
TABLE II

LEFT - SHANNON'S LIMIT (SL) FOR THE CHANNEL WITH SYMMETRIC INTERFERENCE AND THE DYNAMICAL (DT) AND THERMODYNAMICAL (TT) TRANSITIONS FOR DIFFERENT INTERFERENCE VALUES. RIGHT - DT AND TT FOR VARYING NUMBERS OF RELAYS.

\begin{tabular}{|c|c|c|c||c|c|c|}
\hline \multicolumn{3}{|c||}{ Interference } & \multicolumn{3}{c|}{ Relays } \\
\hline \hline$\epsilon$ & SL & DT & TT & $P$ & DT & TT \\
\hline \hline 0.2 & 3.33 & 1.09 & 1.16 & 1 & 1.28 & 1.84 \\
0.4 & 3.42 & 1.41 & 1.65 & 2 & 1.44 & 2.00 \\
0.6 & 3.99 & 1.72 & 2.09 & 3 & 1.51 & 2.07 \\
0.8 & 4.68 & 2.14 & 2.70 & 4 & 1.53 & 2.08 \\
1.0 & 5.63 & 2.63 & 3.33 & 5 & 1.55 & 2.11 \\
& & & & $\infty$ & 1.60 & 2.18 \\
\hline
\end{tabular}

matrix $S$. To make the interpretation of results more transparent, we solve for $L=O=2$. In the symmetric case, transmitters send messages to both receivers while in the asymmetric case, only one transmits to the first receiver while the second transmits to both.

Symmetric Interference: Let $S_{11}=S_{22}=1$ and $S_{12}=S_{21}=\epsilon$, $\epsilon \in(0,1]$. The same scaling as in the MAC case is necessary due to the interference.

Overlaps, entropy and energy are qualitatively the same as before. The threshold noise versus $\epsilon$ is shown in table II (left). Although counterintuitive, the resilience against noise increases with the interference. This occurs because, for joint detection, the increased interference provides more information about the other transmitters.

For large $O$ or $L$ with constant load $\alpha \equiv L / O$, the results should approach those obtained for a large number of users for the single transmitter and MAC, respectively. We expect the results to cross from a MAC $(\alpha>1)$ to a single transmitter-like behaviour $(\alpha<1)$.

Asymmetric Interference: This realisation is highly relevant to cases where receivers are distributed at random and experience different noise levels, for instance, in sensor networks. The interference takes the form $S_{11}=S_{22}=1, S_{12}=\epsilon$ and $S_{21}=0, \epsilon \in(0,1]$. In this case, the scaling $L^{-1 / 2}$ appears only in the first receiver, as it is the only receiver affected by the interference.

The top plot of fig. 3 shows the overlaps of both messages for $\epsilon=1.0$, with squares/circles representing the result of BP averaged over 10 parity-check matrices and 100 codewords with $M=2000$. Interestingly, they behave significantly differently in spite of the joint decoding; a striking feature of this case. Both overlaps are 1 up to the point where the first (upper line) exhibits a dynamical transition marking the practical threshold for the system. This point can be identified as the point where the entropy (bottom right) becomes negative. It is very far from Shannon's limit (dotted line) $\sigma^{2} \approx 7.56$; which is explained by the additional metastable states introduced by the asymmetric interference. Note that the first overlap undergoes a dynamical transition before the second (bottom line) despite of the fact that the messages are decoded jointly. The second message overlap shows an unexpected sudden increase when the first one drops to sub-optimal levels. This may be understood by examining the average overlap, which 

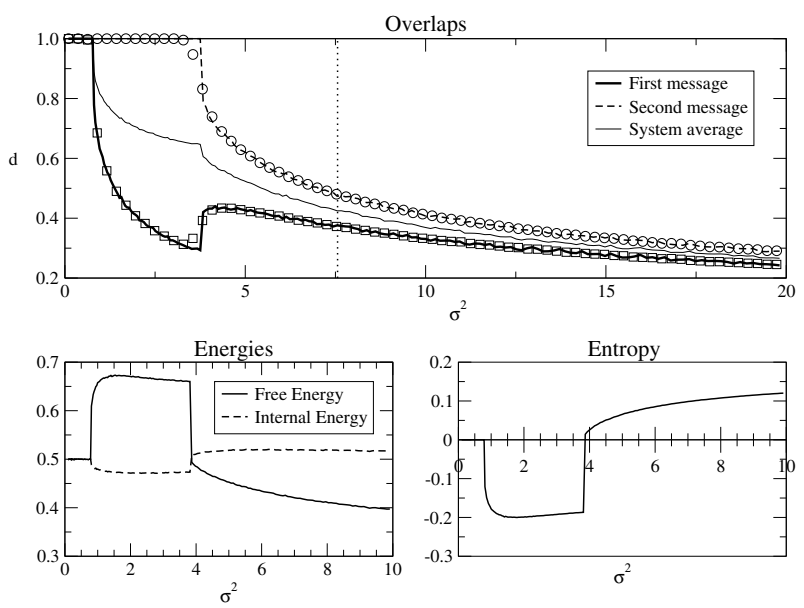

Fig. 3. Asymmetric interference. The upper plot shows the overlap for the first (upper line) and second (bottom line) messages and the average overlap (middle line), with squares and circles obtained by BP. The dotted line is Shannon's limit. At the bottom, we show the free (continuous) and internal (dashed) energies (left) and the entropy (right).

undergoes a second transition at this point, but continues to decrease monotonically: the system as a whole has a certain amount of retrievable information which decreases with the noise. This shows that the distribution of information is highly non-trivial and that for many users, the thermodynamical transition is determined mostly by the weakest node (the highest interference) and may lead to practical limits very far from Shannon's bound.

\section{RElay ARray}

A relay array (fig. 4) is a multiple-unit generalisation of the relay channel [17]. A codeword $\mathbf{t}$ is sent to $P$ relays, corrupted by global AWGN $\mathbf{n}_{0} \sim \mathcal{N}\left(0, \sigma_{0}^{2}\right)$ and local independent AWGNs $\mathbf{n}_{k} \sim \mathcal{N}\left(0, \sigma_{k}^{2}\right)$. Each relay processes a message $\boldsymbol{\rho}_{k}$, encodes the information in $\boldsymbol{\theta}_{k}$ and sends it to the final receiver, which gets their summation plus a direct message (corrupted also by $\left.\mathbf{n}_{0}\right)$ with this sum subject to an AWGN $\boldsymbol{\nu} \sim \mathcal{N}\left(0, \sigma^{2}\right)$. This non-linear case of the matrix channel with $L=O=1$ is given by

$$
\begin{gathered}
t_{\mu}=a t_{\mu}+\sum_{k=1}^{P} b_{k} \theta_{k \mu}+n_{0 k}, \\
\theta_{k \mu}=\phi_{k}\left(\boldsymbol{\rho}_{k}\right), \quad \text { and } \boldsymbol{\rho}_{k}=c_{k} \mathbf{t}+\mathbf{n}_{k}+\mathbf{n}_{0},
\end{gathered}
$$

with relative gains $a, b_{k}$ and $c_{k}$, where each $\phi_{k}$ defines the processing of information by the $k$-th relay.

As the above Hamiltonian is invariant to the gauge transformation $r_{\mu} \rightarrow \gamma_{\mu} r_{\mu}, t_{\mu} \rightarrow \gamma_{\mu} t_{\mu}$, where $\gamma$ obeys the parity-check constraints, at $\beta=1$ the RS solution is exact.

For $P=1$ we have the relay channel. In the classical relay channel (CRC) [18], the relay messages $\boldsymbol{\theta}_{k}$ 's are only allowed to depend on the symbols received prior to the current time, $\theta_{k \mu}=\phi_{k}\left(t_{1}, \ldots, t_{\mu-1}\right)$, modelling the fact that the relay takes some time to process the data. If the time delay in the direct transmission is much longer than to the relays, one can allow a dependence on the present symbol as well, $\theta_{k \mu}=\phi_{k}\left(t_{1}, \ldots, t_{\mu}\right)$. This is called the relay-without-delay (RWD) [19]. When there

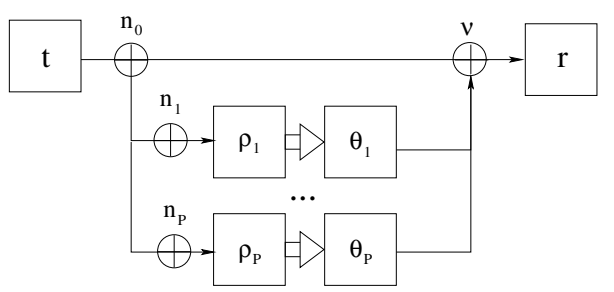

Fig. 4. $P$-component relay array. The transmitter sends a codeword $\mathbf{t}$ to the final receiver and to each relay. The relays receive $\boldsymbol{\rho}_{k}$, the original codeword corrupted by the AWGNs $\mathbf{n}_{0}$ and $\mathbf{n}_{k}$ 's and send to the final receiver the vectors $\boldsymbol{\theta}_{k}$. The final receiver receives the original codeword summed with all relay messages $\boldsymbol{\theta}_{k}$ corrupted by the AWGNs $\mathbf{n}_{0}$ and $\boldsymbol{\nu}$.

is no direct transmission, the restriction to all but the last received symbol is even unnecessary. We focus on a Demodulate and Forward strategy where the relays have incomplete information about the encoding, decoding the message using a uniform prior for the codeword and sending the MPM estimate $\theta_{k \mu}=\operatorname{sgn}\left(\rho_{k \mu}\right)$ to the final receiver.

Relay Channel: We can compare our results with those of [19] by analysing the RWD with $\mathbf{n}_{0}=0, \sigma_{1}^{2}=\eta \sigma^{2}, a=b_{1}=1$ and $c_{1}=\left(1+\sigma^{2}\right)^{-1 / 2}$. Numerical results are given in Fig. 5 for $\eta=0.1$. The vertical line is Shannon's limit $\sigma_{S L}^{2} \approx 8.79$. The dashed curve is an AWGN channel with noise level $\sigma^{2}$ and the continuous one is the RWD. The improvement in the practical limit for error-free communication (the highest noise level for which $d=1$ ) is clear. However, the distance between the dynamical transition $\sigma_{d}^{2} \approx 2.22$ and Shannon's limit is greater than in the AWGN channel [20]. Further calculations point to the expected result that decreasing the noise level $\sigma_{1}^{2}$ brings $\sigma_{d}^{2}$ closer to Shannon's limit. Note that this strategy does not use the full potential of the relay and LDPC decoding in the relay must improve the performance. As noise increases the channel becomes similar to the AWGN channel as the additional information provided by the relay becomes negligible. The inset shows that the dynamical and thermodynamical transitions decrease with $\eta$ but become closer to each other, approaching the matching asymptotic values of the simple AWGN channel as the relay contribution fades away. The entropy is qualitatively the same as in the MIMO case, becoming negative at the dynamical transition and positive again beyond the thermodynamical transition.

We now use a setup equivalent to the one in [18] with $\mathbf{n}_{1}=0, a=c_{1}=b_{1}=1$ and $\sigma_{0}^{2} \equiv \lambda \sigma^{2}$, and $\lambda>0$. Fig. 6 compares the transition levels of a RWD to Shannon's limit for the CRC. Although the practical decoding line (dynamical transition) always falls below Shannon's limit for the CRC, the thermodynamical transition goes above it at higher $\lambda$. This shows that the capacity of the RWD is higher than the CRC here and quantifies the gain in allowing the dependence on the current transmitted symbol for the practical LDPC coding. Although this seems an insignificant modification in the infinite block length limit, it gives relevant extra information which facilitates more efficient retrieval at the final receiver. The insight gained is that for the RWD and large $\lambda$, the relay transmission $\theta_{1 \mu}$ is correlated with the original codeword 


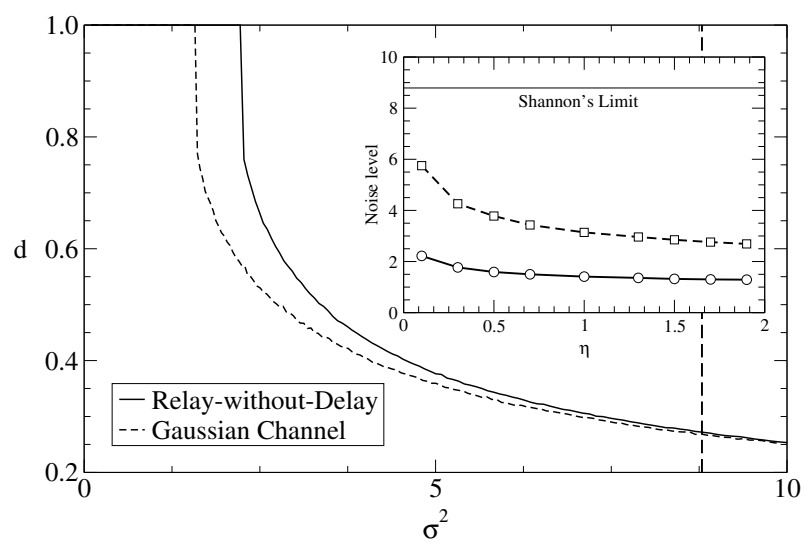

Fig. 5. Overlap for the RWD with $\eta=0.1$ (continuous curve) and for the AWGN channel (dashed curve). The vertical line is Shannon's limit for the RWD [19]. In the inset, the continuous/dashed line shows the dynamical/thermodynamical transitions versus $\eta$. The horizontal line is Shannon's limit.

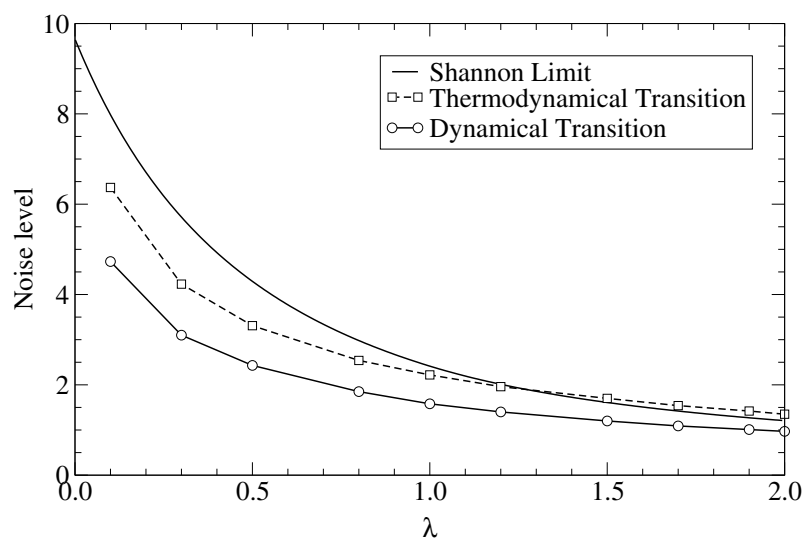

Fig. 6. The continuous/dashed line shows the dynamical/thermodynamical transition for the RWD versus $\lambda$. The continuous line without marked symbols is Shannon's limit for a CRC with the same noise levels and transmission powers.

$t_{\mu}$, which is not the case in the CRC; this allows for an improvement in the information extraction at the receiver.

For $P \rightarrow \infty$, the central limit theorem gives analytical results provided we introduce a $1 / P$ scaling in the summation over relay messages to guarantee a consistent order. Table II (right) compares the dynamical and thermodynamical transitions for $P=1, \ldots, 5$ and the result for $P \rightarrow \infty$ at the same noise level for all relays. The transitions approach the $P \rightarrow \infty$ solution already at $P=5$, making this approximation highly applicable.

\section{CONCLUSION}

We presented a general formulation of vector channels and analysed special cases by methods of statistical mechanics under LDPC coding. We provided solutions for the linear MIMO channel and non-linear relay array. The results obtained coincide with BP solutions up to the thermodynamical transition point and give insight into the information transfer between sender and receiver, including some unexpected ef- fects for the case of asymmetric interference. The approach provides added insight as to the nature and reasons for the various transition points as well as theoretical results that cannot be obtained otherwise (e.g., from DE). While we have concentrated on limited scenarios, we believe that the methods and formulation presented offer a promising alternative to the information theory methodology which had limited success in dealing with multi-user communication systems.

\section{ACKNOWLEDGMENT}

Support from Evergrow, EU-IP 1935 and EPSRC E049516 is acknowledged.

\section{REFERENCES}

[1] H. Nishimori, Statistical Physics of Spin Glasses and Information Processing. Oxford, UK: Oxford University Press, 2001.

[2] T. Tanaka, "A statistical-mechanics approach to large-system analysis of CDMA multiuser detectors," IEEE Trans. Inf. Theory, vol. 11, pp. 2888-2910, 2002.

[3] K. Nakamura, Y. Kabashima, R. Morelos-Zaragoza, and D. Saad, "Statistical mechanics of broadcast channels using low density parity check codes," Phys. Rev. E, vol. 67, p. 036703, 2003.

[4] N. Sourlas, "Spin-glass models as error-correcting codes," Nature, vol. 339, pp. 693-695, 1989.

[5] Y. Kabashima and D. Saad, "Statistical mechanics of low-density parity check codes," J. Phys. A., vol. 37, pp. R1-R43, 2004.

[6] R. Gallager, "Low density parity check codes," IRE Trans. Inf. Theory, vol. IT-8, pp. 21-28, 1962.

[7] T. Richardson, A. Shokrollahi, and R. Urbanke, "Design of capacityapproaching irregular low-density parity-check codes," IEEE Trans. Inf. Theory, vol. 47, pp. 619-637, 2001.

[8] T. Richardson and R. Urbanke, "The capacity of low-density parity check codes under message-passing decoding," IEEE Trans. on Info. Theory, vol. 47, pp. 599-618, 2001.

[9] D. MacKay, Information Theory, Inference and Learning Algorithms. Cambridge, MA: Cambridge University Press, 2003.

[10] R. Vicente, D. Saad, and Y. Kabashima, "Low density parity check codes: A statistical physics perspective," in Advances in Imaging and Electron Physics, P. Hawkes, Ed. USA: Academic Press, 2002, vol. 125, pp. 232-353.

[11] Y. Iba, "The Nishimori line and Bayesian statistics," J. Phys. A, vol. 32, pp. 3875-3888, 1999.

[12] R. C. Alamino and D. Saad, "Statistical mechanics analysis of ldpc coding in mimo gaussian channels," J. Phys A: Math. Theor., vol. 40, pp. 12 259-1279, 2007.

[13] Rev. E, vol. 76, 2007.

[14] S. Franz, M. Leone, A. Montanari, and F. Ricci-Tersenghi, "Dynamic phase transition for decoding algorithms," Phys. Rev. E, vol. 66, p. 046120, 2002

[15] G. Migliorini and D. Saad, "Finite-connectivity spin-glass phase diagrams and low density parity check codes," Phys. Rev. E, vol. 73, p. 026122,2006

[16] T. Tanaka and D. Saad, "A statistical-mechanical analysis of coded cdma with regular LDPC codes," in Proceedings 2003 IEEE International Symposium on Information Theory, Yokohama, Japan, 2003, p. 444.

[17] T. M. Cover and J. Thomas, Elements of Information Theory. New York, NY: John Wiley \& Sons, 1991.

[18] T. M. Cover and A. A. El-Gamal, "Capacity theorems for the relay channel," IEEE Trans. Inf. Theory, vol. 25, pp. 572-584, 1979.

[19] A. El-Gamal and N. Hassanpour, "Relay-without-delay," Proc. Int. Symposium on Inf. Theory, ISIT 2005, pp. 1078-1080, 2005.

[20] T. Tanaka and D. Saad, "Typical performance of low-density paritycheck codes over general symmetric channels," J. Phys. A, vol. 36, pp. 11 143-11 157, 2003. 